\title{
Study on the Characterization Method of Ultrasonic Cavitation Field based on the Numerical Simulation of the Amplitude of Sound Pressure
}

\author{
Tangfei Tao ${ }^{1, *}$, Jinmin Zhao ${ }^{2}$ and Wei Wang ${ }^{2}$ \\ ${ }^{1}$ Key Laboratory of Education Ministry for Modern Design \& Rotor-Bearing System, Xi'an Jiaotong University, Xi'an, China \\ ${ }^{2}$ School of Mechanical Engineering, Xi'an Jiaotong University, Xi'an, China
}

\begin{abstract}
In order to meet the visualization requirements of the cavitation field distribution in the ultrasonic cavitation reactor, a method based on the numerical simulation of the amplitude of sound pressure is proposed. In order to verify that the amplitude of sound pressure plays a decisive role in cavitation effect, the dynamic equation of a single cavitation bubble is established, and the influence law of the amplitude of sound pressure on cavitation motion is analyzed in principle; then, the three-dimensional model of the selfbuilt drum type ultrasonic cavitation reactor is built using the finite element software COMSOL Multiphysics, and the amplitude distribution of the sound pressure at the longitudinal section is obtained when the liquid height was $25 \mathrm{~mm}, 60 \mathrm{~mm}$ and $90 \mathrm{~mm}$. Through the comparison of aluminum foil corrosion experiments, it shows that the numerical simulation method based on the amplitude of sound pressure can accurately characterize the distribution area of ultrasonic cavitation field, which overcomes the disadvantage of time-consuming and labor-consuming in the traditional measurement method of cavitation field distribution, and lays a foundation for the study of the distribution law of ultrasonic cavitation field.
\end{abstract}

\section{Introduction}

Cavitation is essentially a series of processes in which the bubble core in a liquid expands, compresses and collapses under the alternate periodic fluctuation of ultrasonic wave. When a bubble collapses, its temperature can reach over several thousand K [1], and its pressure can reach hundreds or even thousands of standard atmospheric pressure [2]. However, cavitation phenomenon does not always happen, only when the amplitude of sound pressure reaches the cavitation threshold can cavitation effect be caused. The cavitation threshold is the smallest ultrasonic amplitude that can make the cavitation phenomenon happen in the liquid medium [3]. With the increasing demand for visualization of the distribution of ultrasonic cavitation field, in 2008, Mandroyan et al. [4] photographed the cavitation area in the sonochemical reactor by using the laser tomograph technology, and truly characterized the cavitation process of the whole area. It is not only costly to characterize the cavitation area by photographing technology, but also strict for the requirement of experimental environment, which is not conducive to the promotion and use. In 2016, Guo Xuan et al. [5] used aluminum foil cavitation experiment to study the influence of ultrasonic frequency on cavitation region. Although the cost of aluminum foil cavitation experiment is relatively low, it has the disadvantage of time-consuming and labor-consuming. Liu Liyan et al. [6] used hydrophone to scan point by point to collect the acoustic field information in the whole plane, and extracted cavitation energy information through signal processing technology. The hydrophone method is helpful for the quantitative study of ultrasonic cavitation field, however, the cost of hydrophone is relatively high and it also interferes with the experimental conditions.In recent years, people also tried to use computer technology such as computational fluid dynamics, finite element method, time-domain finite difference method and other methods to calculate and simulate the sound field [7-8]. For example, Servant et al. used CFD software to simulate the pressure field and cavitation bubble gas holdup field of the sonochemical reactor [7]. Using computer simulation analysis can get the important reference information of sound field pressure and fluid velocity in each position, which greatly shortens the time period, but the simulation process needs to be further improved, for example, the simulation of the excitation process of piezoelectric transducer needs to be added in the simulation process.

In this paper, firstly, the dynamic equation of a single cavitation bubble is derived by analysing its motion process; then, the acoustic-piezoelectric-electric coupling model is established by the finite element software COMSOL Multiphysics, which has the advantage of multi physical field coupling; finally, the amplitude distribution of sound pressure at different liquid level is compared with the experimental results of cavitation corrosion of aluminum foil. 


\section{Numerical calculation of cavitation equation}

\subsection{Dynamic equation of ultrasonic cavitation bubble}

There are inevitably some bubbles in the liquid, assuming that the temperature of the liquid is constant, and one of these bubbles is studied, which is always spherical in the process of movement, and the partial pressure of the steam in the bubble is equal to the steam pressure at the main body temperature. From the point of view of energy conservation and force balance of cavitation bubble, considering the influence of liquid viscosity and surface tension on the bubble, the nonlinear motion process of cavitation bubble can be described as equation (1) [9].

$$
\begin{gathered}
R\left(\frac{d^{2} R}{d t^{2}}\right)+\frac{3}{2}\left(\frac{d R}{d t}\right)^{2}= \\
\frac{1}{\rho}\left[\left(\mathrm{P}_{\infty}+\frac{2 \sigma}{\mathrm{R}_{0}}\right)\left(\frac{\mathrm{R}_{0}}{R}\right)^{3 \mathrm{n}}+P_{b}+\mathrm{P}_{\mathrm{v}}-\mathrm{P}_{\infty}-\frac{2 \sigma}{R}\right]
\end{gathered}
$$

In equation (1), $t$ is the moving time of bubble, $R$ is the instantaneous radius of bubble, $R_{0}$ is the initial radius of bubble, $\rho$ is the density of liquid, $\sigma$ is the surface tension coefficient of liquid, $\mathrm{P}_{\infty}$ is the static pressure of liquid, $P_{b}$ is the sound field pressure, $\mathrm{P}_{\mathrm{v}}$ is the vapor pressure in the bubble, and $\mathrm{n}$ is the multi index, which represents the thermodynamic state of the process. If the energy viscosity loss and the radiation damping caused by the radiation of the liquid medium are considered, the equation (1) evolves into (2) [10]:

$$
\begin{gathered}
R\left(\frac{d^{2} R}{d t^{2}}\right)+\frac{3}{2}\left(\frac{d R}{d t}\right)^{2}= \\
\frac{1}{\rho}\left[\left(\mathrm{P}_{\infty}+\frac{2 \sigma}{\mathrm{R}_{0}}\right)\left(\frac{\mathrm{R}_{0}}{R}\right)^{3 \mathrm{n}}+P_{b}+\mathrm{P}_{\mathrm{v}}-\mathrm{P}_{\infty}-\frac{2 \sigma}{R}\right] \\
-\frac{4 \mu}{\rho \mathrm{R}} \frac{d R}{d t}+\frac{R}{\rho c} \frac{d}{d t}\left[\left(\mathrm{P}_{\infty}+\frac{2 \sigma}{\mathrm{R}_{0}}\right)\left(\frac{\mathrm{R}_{0}}{R}\right)^{3 \mathrm{n}}+P_{b}\right]
\end{gathered}
$$

In equation (2) , $\mathrm{c}$ is the velocity of sound in the liquid, $\mu$ is the viscosity coefficient of the liquid.

\subsection{Dynamic simulation of cavitation bubble}

In the study, water under standard atmospheric pressure is used as liquid medium, $\mathrm{T}_{0}=293.15 \mathrm{~K}, \rho=$ $998.3 \mathrm{~kg} / \mathrm{m}^{3}, \quad \sigma=0.0728 \mathrm{~N} / \mathrm{m}, \mu=0.001 \mathrm{~kg} /(\mathrm{m}$. $\mathrm{s}), \mathrm{c}=1483 \mathrm{~m} / \mathrm{s}$. In addition, the multi index is $4 / 3$, indicating that the cavitation bubble movement is an isothermal process. And other parameters are: $\mathrm{P}_{\infty}=$ $1.013 \times 10^{5} \mathrm{~Pa}, \mathrm{P}_{\mathrm{v}}=2340 \mathrm{~Pa}$. The ultrasonic excitation is sine wave:

$$
P_{b}=-\mathrm{P}_{\mathrm{a}} \sin (2 \pi \mathrm{ft})
$$

The dynamic equation of cavitation bubble is solved by using Runge-Kutta numerical iteration method in MATLAB software, the initial conditions are: $P_{a}=$
$1.5 \times 10^{5} \mathrm{~Pa}, \mathrm{f}=28 \mathrm{KHz}, \quad t=0, \quad R=\mathrm{R} 0=6 \mu \mathrm{m}$, $d R / d t=0$. It can be seen from the Fig. 1 that under the influence of ultrasound, the cavitation bubble expands to 9-10 times of the initial radius in a sound pressure cycle of ultrasound, and then rapidly shrinks to 0 after reaching the maximum radius, which is the collapse state, and finally the vibration phenomenon will occur.

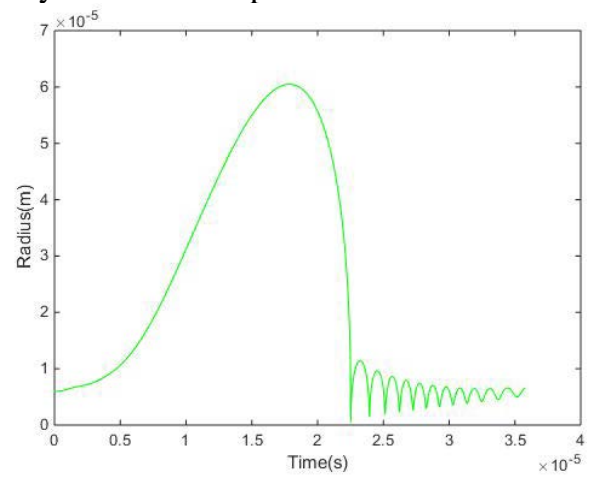

Fig. 1. Curve of cavitation bubble radius with time.

Bubble expansion is due to the fact that the ultrasonic sine wave first enters into a negative half cycle, the pressure in the bubble is greater than the external ultrasonic pressure, so that the cavitation bubble continues to absorb energy, and the radius gradually increases, and the growth rate of the radius will accelerate with the increase of the external sound intensity, and then the growth rate will slow down due to the decrease of the sound intensity; when the ultrasonic sine wave enters the positive half period, the radius of cavitation bubble basically reaches the maximum value, and then with the increase of sound intensity, the radius of cavitation bubble rapidly shrinks to the minimum value; finally, the cavitation bubble enters the shock stage, because when the cavitation bubble is at the minimum radius, the pressure in the bubble is still very large in a short time, resulting in the radius of the bubble increasing to the second maximum point, because at this time the ultrasonic sine wave is still in the positive half cycle, the bubble will be compressed again, and then the process will be repeated all the time. Until the end of this ultrasonic sine wave period, the bubble radius will tend to the initial radius, and wait for the arrival of the next ultrasonic sine wave negative half cycle.

\subsection{Influence of ultrasonic pressure amplitude on cavitation effect}

In order to explore the influence of ultrasonic pressure amplitude on the cavitation bubble movement process, it is known that the original sound pressure amplitude: $\mathrm{P}_{\mathrm{a}}=1.5 \times 10^{5} \mathrm{~Pa}$, keep other quantities unchanged, different ultrasonic pressure amplitude is selected. In Fig. 2 a), by comparing the bubble radius curve with the sound pressure amplitude of $1 \mathrm{P}_{\mathrm{a}}$, it can be found that the larger the ultrasonic pressure amplitude is, the larger the maximum radius the cavitation bubble can reach, and the later the bubble will enter the stage of collapse and shock. Because when the ultrasonic frequency is constant, the larger the amplitude of ultrasonic pressure is, the more 
energy the cavitation bubble absorbs in the negative half cycle of the sound pressure, the longer the expansion time, the larger the maximum radius the bubble can reach, and the later compression and collapse of the bubble are delayed.

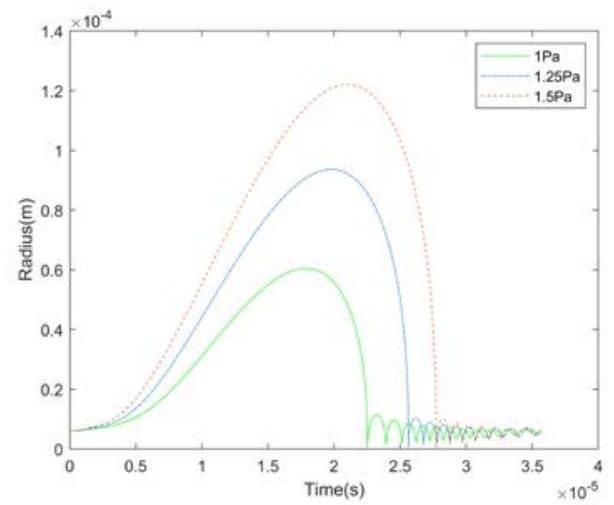

a)

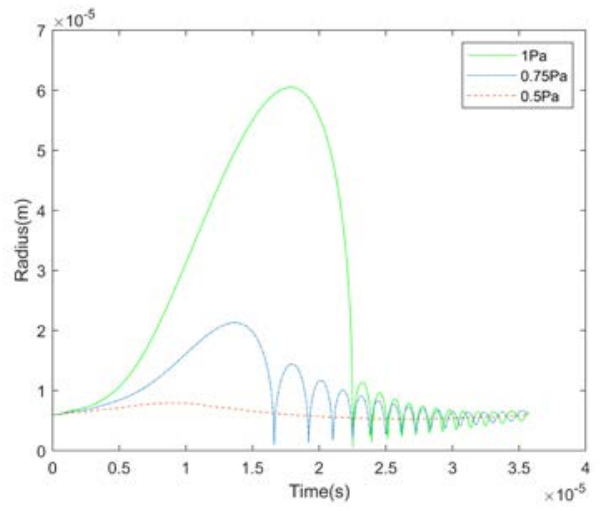

b)

Fig. 2. a) Curve of spatiotemporal bubble radius with sound pressure amplitude of $1.5 \mathrm{P}_{\mathrm{a}}, 1.25 \mathrm{P}_{\mathrm{a}}, 1 \mathrm{P}_{\mathrm{a}}$. b) Curve of spatiotemporal bubble radius with sound pressure amplitude of $1 \mathrm{P}_{\mathrm{a}}, 0.75 \mathrm{P}_{\mathrm{a}}, 0.5 \mathrm{P}_{\mathrm{a}}$.

In Fig. 2 b), by comparing the bubble radius curve with the sound pressure amplitude of $1 \mathrm{P}_{\mathrm{a}}$, when the amplitude of super sound pressure is taken as three quarters of the original value, the maximum value that the bubble radius can reach will be relatively reduced, but there will be expansion, compression, collapse and vibration in the process of bubble movement; however, when the amplitude of sound pressure is taken as half of the original value, bubble radius changes little with time, without expansion, compression and other phenomena, because the amplitude of ultrasonic pressure is small or lower than the threshold value of ultrasonic cavitation, the energy absorbed by cavitation bubbles from the negative half cycle of ultrasonic sine wave makes the degree of self-expansion smaller or not enough to make self-expansion, which makes cavitation difficult to occur.

\section{Ultrasonic cavitation experiment and simulation analysis}

\subsection{Cavitation test device}

In order to verify the correlation between the distribution area of the amplitude of the sound pressure in the reactor and the area of the cavitation intensity, a self-made cylindrical ultrasonic cavitation experimental device is made. The wall thickness of the cylindrical reactor is $3 \mathrm{~mm}$, the inner diameter is $52 \mathrm{~mm}$, and the total height is $155 \mathrm{~mm}$. An ultrasonic transducer is bonded in the middle of the bottom, its frequency is $28 \mathrm{kHz}$, and the rated power is $60 \mathrm{~W}$. The schematic diagram of the experimental device is shown in Fig. 3. After the power supply of the ultrasonic generator is connected, the electric energy is converted into the ultrasonic signal by the transducer, and the sound wave is transmitted to the liquid medium through the cylindrical reactor. PLC can set delay program to ensure that the time of each cavitation experiment is 90s, and data recorder can measure the temperature to ensure that the temperature in the liquid medium is the same at the beginning of each cavitation experiment.

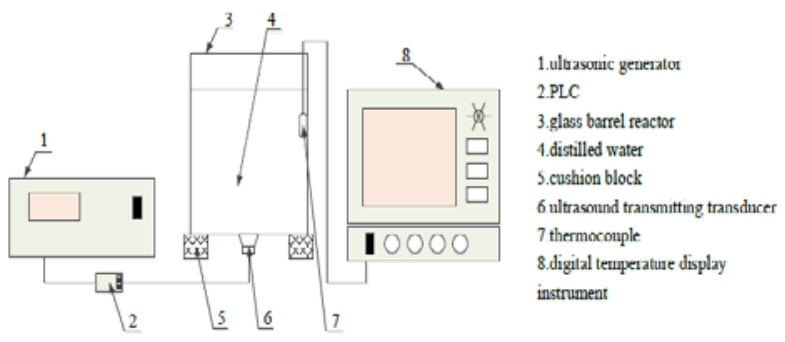

Fig. 3. Cylindrical ultrasonic cavitation reactor.

Before the experiment, different volumes of distilled water were successively injected into the cylindrical reactor, at the same time, the aluminum foil with a flat surface and a thickness of twenty microns is fixed on the processed frame. The width of the frame is the same as the diameter of the reactor, and the height is flush with the liquid level, and then it is placed at the maximum longitudinal section of the reactor. When the power supply is turned on, the aluminum foil will be corroded due to the cavitation effect produced in the liquid. With the extension of the cavitation time, there will be pits and holes of different sizes on the surface of the aluminum foil. After the experiment, the aluminum foil will be dried and the image scanning will be carried out, and the cavitation corrosion distribution area of this plane can be observed from the image.

\subsection{Simulation process based on COMSOL Multiphysics software}

In order to simulate the excitation process of the ultrasonic transducer in the cavitation experimental device reasonably and to consider that the acoustic wave propagation will be reflected and refracted by the reactor wall, at the same time, considering the obvious characteristics of the solid-liquid interface in the reactor, physical fields such as solid mechanics and static electricity are added to the acoustic module of the finite element software COMSOL Multiphysics. Then according to the size of the experimental device, the acoustic-piezoelectric-electric coupling model is built in 
the ratio of 1 to 1 , as shown in Fig. 4, in which the transducer is approximately treated as a cylinder shape. The finite element model includes three different structures: transducer, reactor and liquid. Their materials are selected from the material library built in simulation software. The transducer in this model is PZT-4 type, the reactor material is $\mathrm{SiO} 2$ (fused quartz), the liquid material is liquid water, and the boundary conditions of hard sound field are set at the interface of convection and solid, at the same time, the boundary conditions of hard sound field are set at the interface of liquid-solid interface.

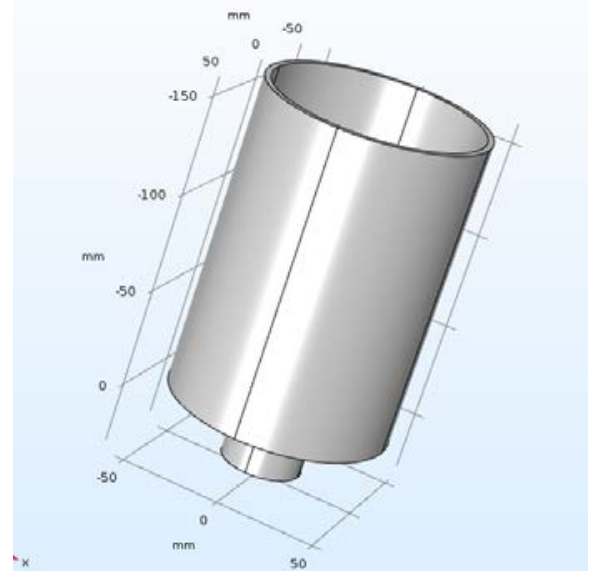

Fig. 4. Three dimensional finite element model.

Different structures need different mesh forms, the transducer uses mapping and sweeping methods to mesh, and the default polarization direction is along the z-axis; the reactor and liquid basically use free tetrahedron mesh method, it should be noted that the mesh size of the liquid needs to be set to one fifth of the wavelength.

\section{Results and discussion}

\subsection{Cavitation corrosion test results}

In order to increase the number of comparison with the simulation results and the number of cavitation corrosion tests, under the same external factors such as temperature, etc., the aluminum foil were put into the reactor longitudinal section with the liquid level of 25 $\mathrm{mm}, 60 \mathrm{~mm}$ and $90 \mathrm{~mm}$ successively for cavitation test, and the aluminum foil images were scanned after each test. The images after the test is shown in Fig. 5.

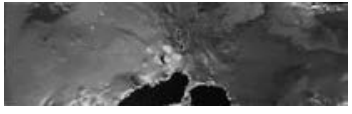

a)

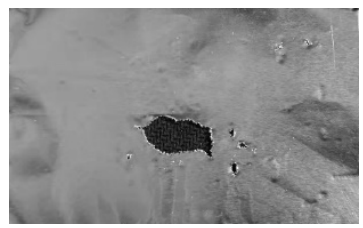

b)

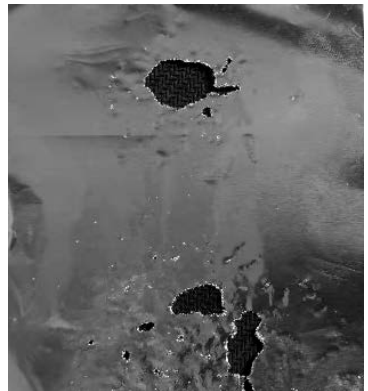

c)
Fig. 5. a) Corrosion image of aluminum foil with liquid level of $25 \mathrm{~mm}$. b) Corrosion image of aluminum foil with liquid level of 60mm. c) Corrosion image of aluminum foil with liquid level of $90 \mathrm{~mm}$.

On the vertical surface, it can be found that the cavitation corrosion area is concentrated near the central axis. When the liquid level is low, the cavitation area appears at the bottom of the aluminum foil. When the liquid level rises to $60 \mathrm{~mm}$, the cavitation area rises to the middle of the aluminum foil. When the liquid level rises to $90 \mathrm{~mm}$, the cavitation area appears at the bottom and the top of the aluminum foil. Small holes and pits appears in other positions of aluminum foil, indicating that these areas are also affected by cavitation corrosion, but the degree is relatively small.

\subsection{Simulation results and analysis discussion}

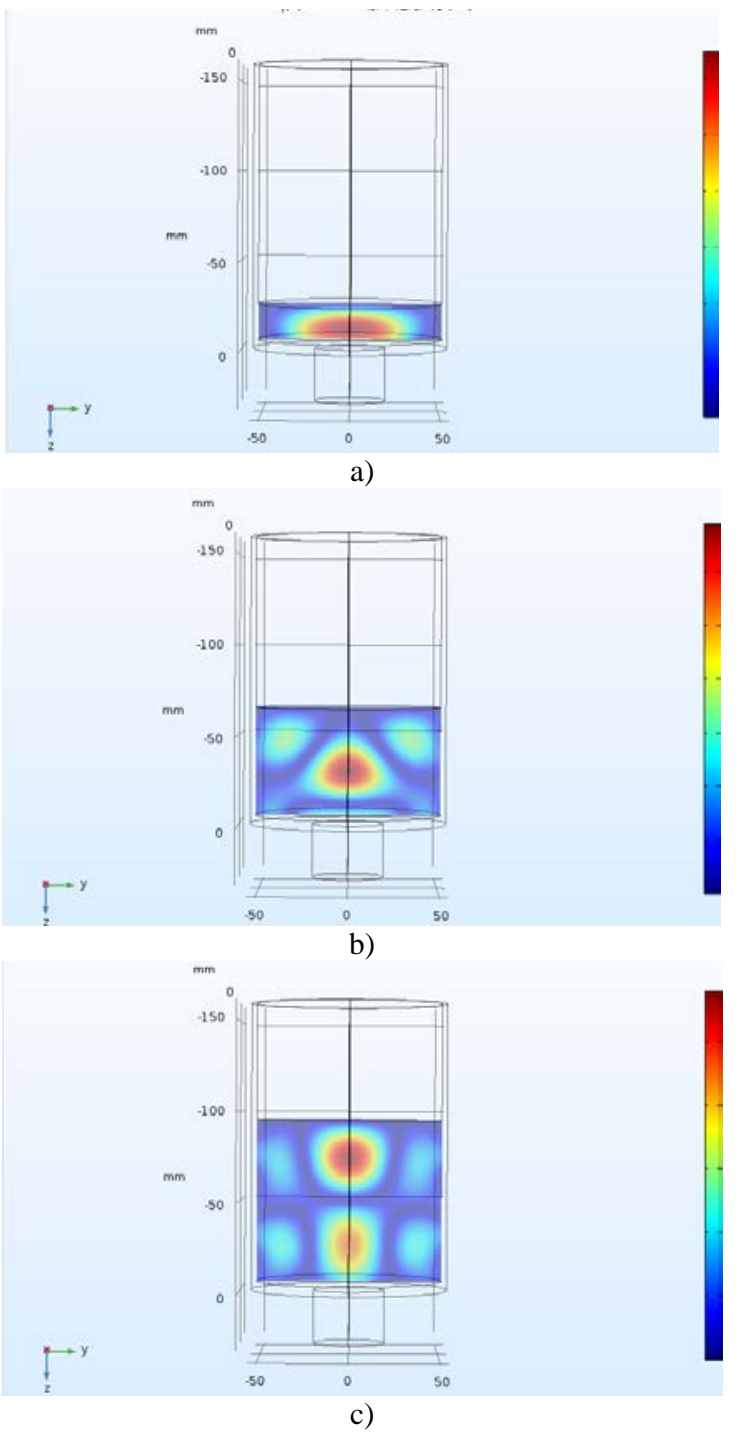

Fig. 6. a) Simulation result of sound pressure amplitude with liquid level of $25 \mathrm{~mm}$. b) Simulation result of sound pressure amplitude with liquid level of $60 \mathrm{~mm}$. c) Simulation result of sound pressure amplitude with liquid level of $90 \mathrm{~mm}$. 
When solving the simulation model in the frequency domain, besides setting the frequency to $28 \mathrm{kHz}$, a longitudinal section should be set along the z-axis direction for the liquid area in the simulation model, and the intensity of the sound field in the section should be characterized by the amplitude of sound pressure.

It should be noted that the simulation results only describe the amplitude distribution of sound pressure, From Fig. 6, the distribution of sound pressure amplitude can be observed, in which the red area represents the high amplitude area and the blue area represents the low amplitude area. Comparing the simulation results of sound pressure amplitude with aluminum foil corrosion experiment, it can be found that the area with higher amplitude of sound pressure is indeed the cavitation corrosion area of aluminum foil, while the area with lower amplitude of sound pressure is basically free of cavitation corrosion. Because the direct sound wave excited by the bottom transducer will reflect when it reaches the gas-liquid interface at the top, the reflected wave and the direct wave will be superposed to form the standing wave form of sound wave, so there will be areas with higher amplitude of sound pressure (wave belly) and lower amplitude of sound pressure (wave node) in the reactor. The height of the liquid surface will change the distance of the sound wave propagation, so the height of the liquid surface has an important influence on the distribution of the amplitude of the sound pressure.

\section{Summary}

In this paper, firstly, the influence of ultrasonic pressure amplitude on the cavitation process is analyzed from the microcosmic point of view, and the dynamic equation of cavitation bubble is calculated numerically; then cavitation corrosion test is carried out in the cylindrical cavitation reactor, and the distribution of ultrasonic pressure amplitude calculated by simulation software is compared, and the following conclusions are drawn:

(1)If the amplitude of sound pressure is lower, or even lower than the cavitation threshold, the cavitation phenomenon is difficult to produce.

(2)COMSOL Multiphysics software can be used to simulate the amplitude distribution of sound pressure in the reactor, and the simulation results are basically consistent with the aluminum foil corrosion test results.

(3)The results show that it is of great significance to study the distribution of ultrasonic cavitation field qualitatively by using the method of sound field simulation.

\section{Acknowledgments}

This work was supported by National Natural Science Foundation of China (No. 51575422).

\section{References}

1. Eddingsaas N C, Suslick K S. Light from sonication of crystal slurries, J. Nature. 444(9), 136, (2006)

2. Suslick K S, Neis U. The Chemical Effects of Ultrasound, J. Scientific American. 43(2), 80-86, (1989)

3. Prosperetti A. Physics of Acoustic Cavitation, M. Frontiers in Physical Acoustic, 145-183, (1986)

4. Mandroyan A, Viennet R. Modification of the ultrasound induced activity by the presence of an electrode in a sonoreactor working at low frequencies (20 and $40 \mathrm{kHz}$ ). Part I: active zone visualization by laser tomography, J. Ultrasonics Sonochemistry, 5(3), 1-19(2008)

5. Guo Xuan, Yang Yanling, Li Xing, et al. Measurement and visualization of ultrasonic cavitation field based on MATLAB, J. China Environmental Science, 036(003) , 719-726(2016)

6. Liu, Liyan, Liu Penghong, Yang Yang, et al. Effect of ultrasonic frequency and liquid level on the cavitation field, J. Journal of Tianjin University, 49(8), 802-808(2016)

7. Servant G, Laborde J L. Spatio-temporal dynamics of cavition bubble clouds in a low frequency reactor: comparison between theoretical and experimental results, J. Ultrasonics Sonochemistry, 8(3), 163174(2001)

8. Rhee S H, Kawamura T, Li H Y. Propeller cavitation study using an unstructured grid based Navier-Stoker slover, J. Journal of Fluids Engineering, 127(5), 986-994(2005)

9. Allen, John S, Roy, Ronald A. Dynamics of gas bubbles in viscoelastic fluids. II. Nonlinear viscoelasticity, J. Journal of the Acoustical Society of America, 108(4), 1640-1650(2000)

10. Chen Weizhong, Chen Xi, Lu Meijun, et al. Single bubble sonoluminescence driven by non-simpleharmonic ultrasounds, J. Journal of the Acoustical Society of America. 111(6), 2632-2637(2002) 